\title{
Surface Microscopy Characterizations of Large Size Graphene films Grown by Surface Segregation on Ni and Transferred to $\mathrm{Si} / \mathrm{SiO}_{2}$ Substrate
}

\author{
D. Pandey ${ }^{\mathrm{a}, \mathrm{b}}$, G. Prakash ${ }^{\mathrm{a}, \mathrm{b}}$, Q.Yu ${ }^{\mathrm{d}}$, H. Cao ${ }^{\mathrm{a}, \mathrm{b}}$, L. A. Jauregui ${ }^{\mathrm{b}, \mathrm{c}}$, \\ S. S. Pei ${ }^{\mathrm{d}}$ and Yong P. Chen ${ }^{\mathrm{a}, \mathrm{b}, \mathrm{c}}$ \\ ${ }^{a}$ Department of Physics, Purdue University, West Lafayette, Indiana 47907, USA \\ ${ }^{b}$ Birck Nanotechnology Center, Purdue University, West Lafayette, Indiana 47907, USA \\ ${ }^{c}$ School of Electrical and Computer Engineering, Purdue University, West Lafayette, \\ Indiana 47907, USA \\ ${ }^{d}$ Department of Electrical and Computer Engineering and Center for Advanced \\ Materials, University of Houston, Houston, Texas 77204, USA
}

\begin{abstract}
We report surface microscopy characterizations of large size graphene films (up to $\mathrm{mm}$ ) grown on polycrystalline $\mathrm{Ni}$ foils and transferred to $\mathrm{Si} / \mathrm{SiO}_{2}$. Wrinkles in such films are studied by both atomic force microscopy (AFM) and scanning tunneling microscopy (STM). Local graphitic lattice structures of the films are imaged with atomic-resolution STM and compared with those of the highly ordered pyrolytic graphite (HOPG).
\end{abstract}

\section{Introduction}

Graphene based nanomaterials have generated tremendous interest in the past few years due to their remarkable material properties and application potentials [1,2]. One of the oldest methods to grow high quality graphene layers is chemical vapor deposition (CVD) or associated surface segregation on various metals (see review in Ref. 3), such as Ni. Such graphene layers can be transferred to other substrates $[4,5,6,7]$ and maintain their high quality. Recent works have demonstrated large size, transparent, flexible and conducting graphene films fabricated by such methods. Despite the non-uniform thickness (number of graphene layers) in the samples fabricated so far, such large size graphene films have been shown to have excellent material and electronic properties that may enable many applications [5,6,7]. In this paper, we present structural and surface characterizations of our mm-scale graphene films grown on polycrystalline Ni foils and transferred to $\mathrm{Si} / \mathrm{SiO}_{2}[4,7]$. We use both atomic force microscopy (AFM) and scanning tunneling microscopy (STM) to study the surface morphology, topography and structural integrity of our films at various length scales. We have characterized wrinkles, which are dominant surface features in the films at large scale and suggested to be important for the flexibility [6] of such large films. We also characterize the local graphitic lattice structure of the films, and make comparison with that of highly ordered pyrolytic graphite (HOPG).

\section{Sample Preparation and General Features}

$\underline{\text { Synthesis and transfer of large area graphene films }}$

The large area graphene films used in this study are synthesized by CVD based surface segregation, as described in detail previously [4]. Briefly, graphene layers are segregated from the surface of polycrystalline $\mathrm{Ni}$ foils under the ambient pressure by dissolving 
carbon in Ni at high temperature followed by controlled cooling. Graphene segregation by cooling is a non-equilibrium process that in general strongly depends on the cooling rate, among other parameters [4]. Previously these films, with up to several mm's in size, are analyzed [4] by transmission electron microscopy (TEM), selected area electron diffraction (SAED) and Raman spectroscopy, and shown to contain graphene layers (ranging from as few as 2 to as many as more than 10) with low defect density.

The as-grown graphene films used in this study are separated from Ni substrates by etching the $\mathrm{Ni}$ metal in aqueous $\mathrm{HNO}_{3}$ solution then transferred [7] to $\mathrm{SiO}_{2}$ (thickness $=300 \mathrm{~nm}$ ) on $\mathrm{Si}$ wafers (around $1 \mathrm{~cm}$ by $1 \mathrm{~cm}$ in size). The graphene films we obtained are semi-transparent, but are easily visible with their distinctive color contrast on the $\mathrm{Si} / \mathrm{SiO}_{2}$ substrates. Figure 1 shows optical microscope images of several transferred films. The films are clearly highly non-uniform at such length scale (varying colors mostly corresponding to varying thickness) $[5,6]$. Wrinkles, which are more evident in AFM and STM images as will be seen next, are nonetheless visible on most of the samples even in such relatively low resolution optical microscope images (Fig. 1a, b). These wrinkles, typically tens of nm in height (deduced from AFM and STM images) are the dominant large scale surface morphology and topography features in our films. From Fig. $1 \mathrm{a}$ and $1 \mathrm{~b}$, we can notice the frequent appearance of $\sim 120^{\circ}$ angle between wrinkles, suggesting the wrinkles may form along preferred directions related to the underlying crystalline structure of the graphene film. Other large surface features we observe on relatively more rare occasions include the self-folding of the film as seen in Fig. 1c (dashed arrow). This particular sample also has a gigantic buckling line (solid arrow, Fig 1c) reaching few microns in height. Given the large-scale thickness non-uniformity and surface roughness, it is remarkable that such large size films are still found to have excellent electronic properties (such as ambipolar field effect, carrier mobilities exceeding $2000 \mathrm{~cm} 2 / V s$ and quantum effects in electronic transport) [7].

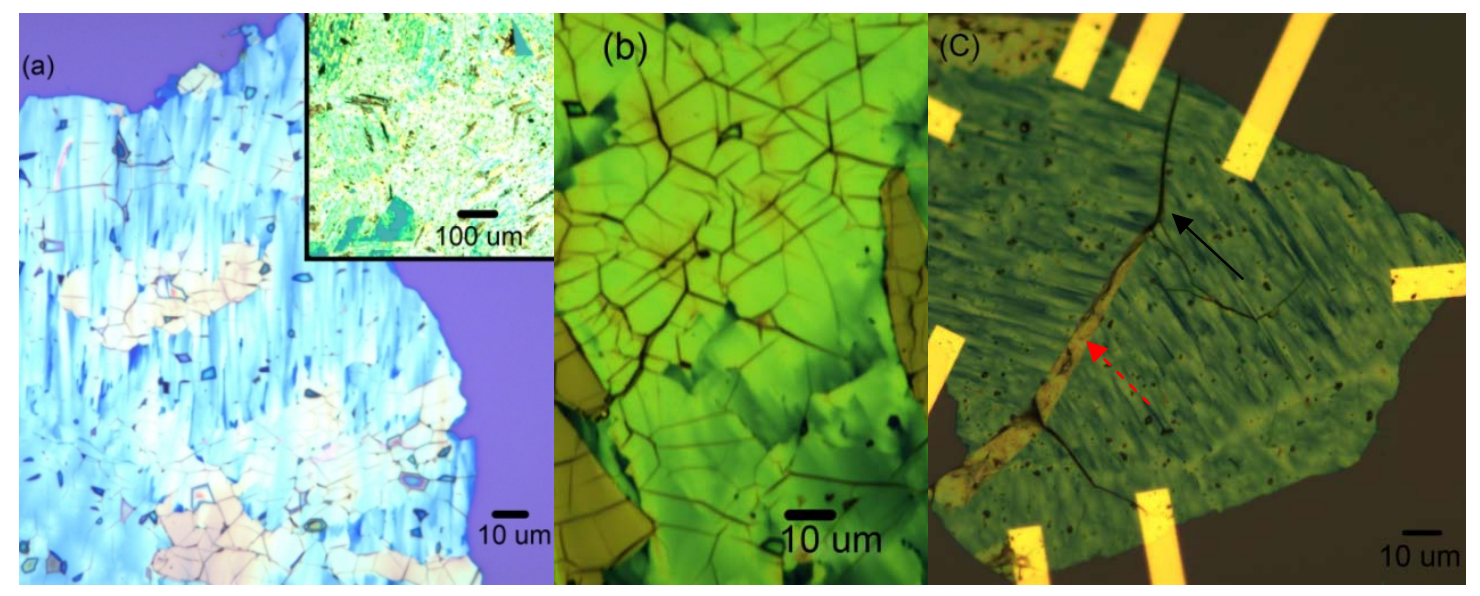

Figure 1. Optical microscope images of graphene films transferred on $\mathrm{Si} / \mathrm{SiO} 2$ substrates.

(a) Image of a sample used in our STM study. The inset shows the image with lower magnification. (b) Image of another similar sample with more evident wrinkles. (c) Another sample showing a particularly large wrinkle (solid arrow) connected with a region (dashed arrow) where the film folds onto itself. The yellow bars are the electrical contacts. 


\section{Experimental Consideration}

Two experimental setups are used to investigate the structural properties of large size graphene films prepared on $\mathrm{Ni}$ by surface segregation and transferred onto $\mathrm{Si} / \mathrm{SiO}_{2}$ substrates. STM experiments are performed under ambient conditions by Nanotech Electronica's Dulcinea Scanning Probe Microscopy (SPM) system. Dulcinea SPM head is housed in a Faraday Cage in a low noise room at Birck Nanotechnology Center, Purdue University. The tip used in the STM study is made of Pt/Ir by cutting with a wire cutter. The gap (bias) voltage is applied to the tip with respect to the sample, which is at ground potential. The STM scans are limited to $4 \mu \mathrm{m} \times 4 \mu \mathrm{m}$ or less. In order to perform STM on the films transferred to insulating $\mathrm{SiO}_{2}$, the upper surface of the graphene film is connected by a thin strip of conductive copper tape to provide the conducting channel. The atomically resolved images from highly oriented pyrolytic graphite (HOPG) are used to calibrate X-Y piezo used in the instrument. The STM images are analyzed using WSxM version 13.0.

Topography and phase contrast AFM images are obtained under ambient condition also by Nanotech Electronica's Dulcinea Scanning Probe Microscopy (SPM) system. The images shown are obtained in intermittent contact mode using microcantilever from Nanosensors (model PPP-NCLR) with a nominal resonance frequency $190 \mathrm{kHz}$ and a nominal spring constant of $48 \mathrm{~N} / \mathrm{m}$. The z-axis of the AFM is calibrated against the step height of highly oriented pyrolytic graphite (HOPG). The $\mathrm{x}, \mathrm{y}$ calibrations are performed using a Micromasch (Model-TGZ01) xyz calibration grating. WSxM software is used to both acquire and analyze the images [8]. The images are scanned at a scanning rate of 0.5 Hz. The AFM scans are generally limited to scan area $12 \mu \mathrm{m} \times 12 \mu \mathrm{m}$ or less.

\section{Results and Discussion}

\section{$\underline{\text { AFM study of as-grown graphene films on Ni surface }}$}

An extensive AFM study is performed on the graphene samples as grown on $\mathrm{Ni}$ substrates using surface segregation. The aim of this study is to understand the morphology and quality of the graphene prepared by surface segregation. AFM image in Figure 2 shows buckling of graphitic layers formed over Ni substrate. The origin of the buckling in the overlay graphene film is believed to be the compressive stress generated in the process of cooling of $\mathrm{Ni}$ substrate from the high temperature $\left(1000{ }^{\circ} \mathrm{C}\right)$ used to dissolve carbon to the room temperature $\left(20^{\circ} \mathrm{C}\right)$. The thermal expansion coefficient (CTE) of $\mathrm{Ni}$ is $\sim 20 \times 10^{-6} \mathrm{~K}^{-1}$ compared to that of graphite $\left(\sim 20 \times 10^{-6} \mathrm{~K}^{-1}\right)[9,10]$ in this temperature range. As $\mathrm{Ni}$ is cooled down from it contracts by $\sim 2 \%$ whereas graphite contracts by only $0.1 \%$. This mismatch in the compressive strain will cause the film to buckle. As can be seen in Figure 2, the buckled wrinkles are often interconnected, diverging from a common point and forming a subtended angle $\theta$. A typical value for the angle $(\theta)$ is statistically determined by measuring the subtended angles between the wrinkles observed in AFM images. Figure 2c (histogram of the angle $\theta$ between wrinkles) shows a preference for the angle near $120^{\circ}$ between major wrinkles. 


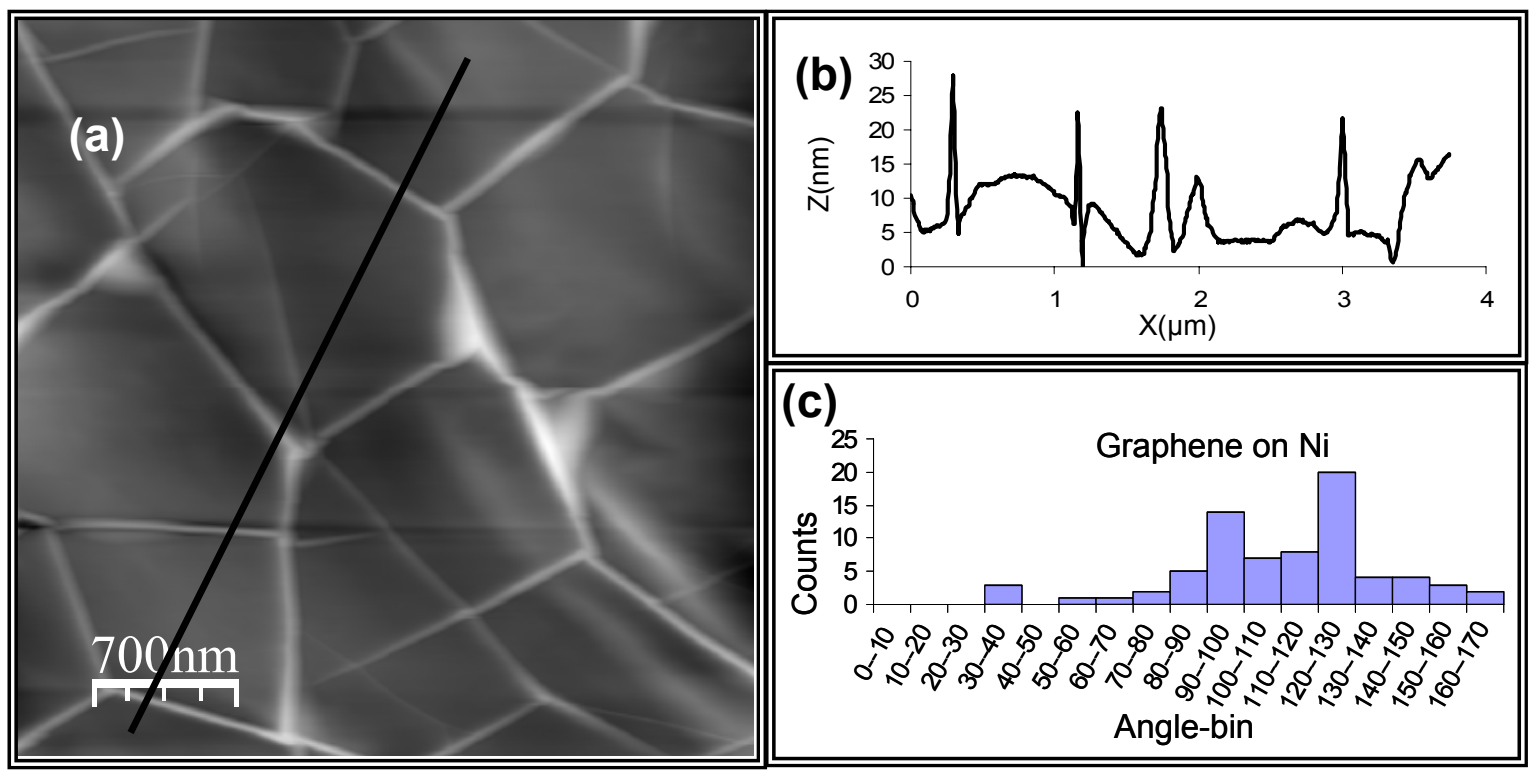

Figure 2. (a) AFM scan of a graphene film over Ni grown by surface segregation. (b) Cross sectional height profile along the line in (a). (c) Histogram (collected from images of multiple regions of the sample) of angles between wrinkles.

$\underline{\text { STM study of graphene films after transferring to } \mathrm{SiO}_{2}} \underline{\text { substrate }}$

Extensive STM study is performed on graphene films after transferring to $\mathrm{Si} / \mathrm{SiO}_{2}$ substrate. STM is chosen to verify that the transferred carbon material after etching the $\mathrm{Ni}$ substrate is graphitic in nature and could conduct electricity. The study ranged from large area topographic imaging to high resolution atomic scale imaging.

Typical large area (4 $\mu \mathrm{m} \times 4 \mu \mathrm{m})$ STM images reveal features that also appear as wrinkles. As shown in Figure 3, STM topography images revealed the surface structure of the graphene film after transferring to the $\mathrm{SiO}_{2}$ substrate, indicating the features that look qualitatively similar to those obtained by AFM of graphene film on Ni substrate. On the other hand, some differences are also noticed between the features in transferred graphene films and those in as-grown films (imaged by AFM). For example, the relatively flat regions of as-grown graphene films on Ni have rms roughness $2.6 \pm 0.5 \mathrm{~nm}$ as compared to $10.1 \pm 0.5 \mathrm{~nm}$ for flat regions of transferred graphene films on $\mathrm{SiO}_{2}$. This is clearly visible in Figure 3(c) at regions 1, 2 and 3 in the form of bulges. Also, many wrinkles seen in transferred films appear to not have as well-defined angular preference as compared to the as-grown films on $\mathrm{Ni}$.

Some of these features may originate from the transfer process. For example, we speculate that as graphene film is transferred on the $\mathrm{SiO}_{2}$ substrate from $\mathrm{HNO}_{3}$ solution, some of the solution is trapped between the film and the $\mathrm{SiO}_{2}$ substrate. As the trapped solution recedes it leaves behind a wrinkled over layered graphene film surface, or bulges the graphene film surface. Figure 3(c) clearly indicates the presence of wrinkles on graphene surface and bulges in region 1,2 and 3 after transferring graphene film on $\mathrm{SiO}_{2}$ substrate. The height of these wrinkles ranges from $\sim 20 \mathrm{~nm}$ to $50 \mathrm{~nm}$. These wrinkles/bulges that may originate from the transfer process clearly form by very 
different mechanisms compared to those on as-grown graphene (formed by differential thermal contraction). Further studies are to be performed to clarify the effect of transfer process on the wrinkles in the graphene films.
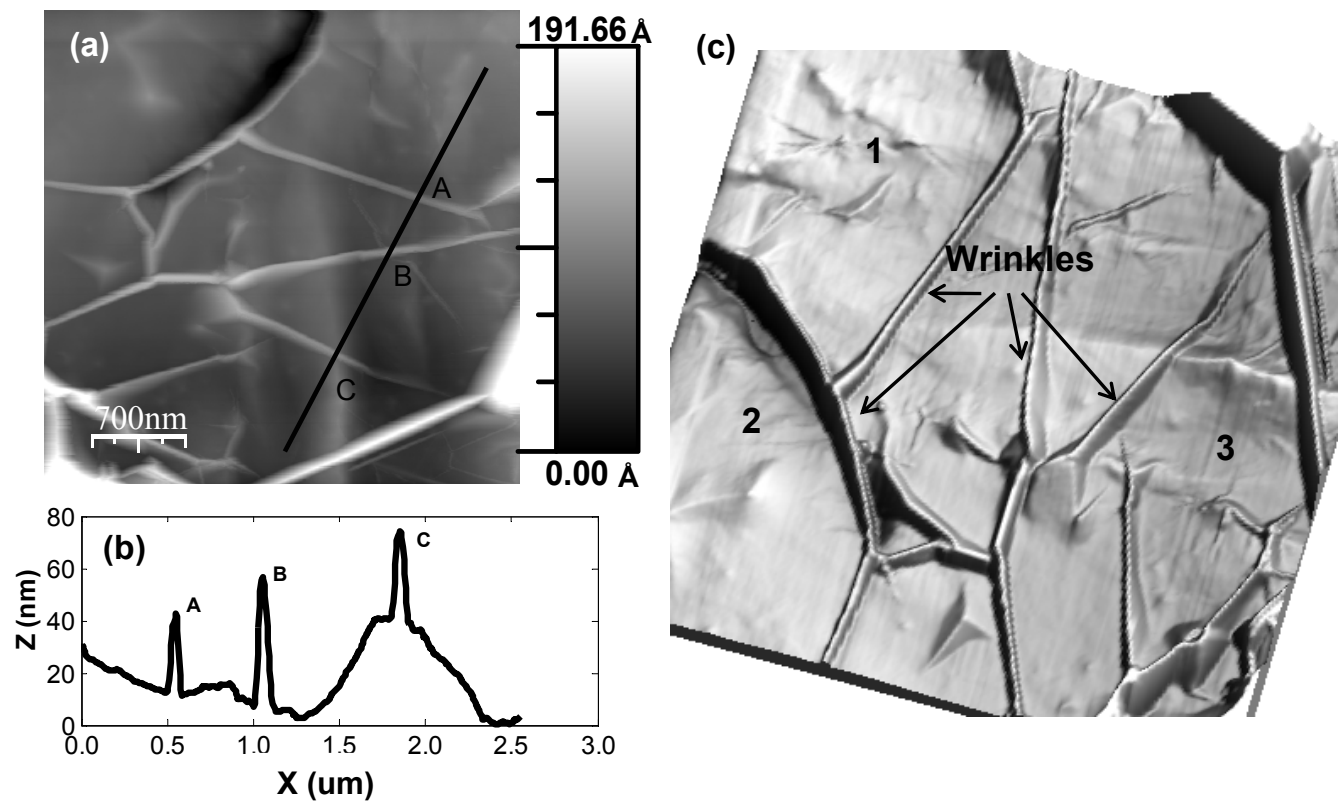

Figure 3. Low resolution STM image of transferred graphene film on $\mathrm{SiO}_{2}$. (a) $2 \mathrm{D}$ image of graphene film indicating wrinkles. (b) Line profile of the selected region indicating the height of the wrinkle and underlying $\mathrm{SiO}_{2}$ substrate. (c) 3D image of Figure 3 (a) clearly indicating wrinkle and three different regions with crumbled features. Some of these features may originate from the transfer process.

Atomically resolved constant current images of clean HOPG are taken to calibrate the piezo scanner. The atomic features are organized in a hexagonal pattern with an atomic spacing of $0.25 \mathrm{~nm}$ [11]. The periodicity of atomic features on HOPG is independent of the scanning rate and bias voltage. Similar constant current studies are then performed on graphene films transferred on $\mathrm{SiO}_{2}$ substrate.

Figure 4 shows a high resolution STM image of a graphene film on $\mathrm{SiO}_{2}$ substrate. Atomically resolved images are obtained between wrinkles on relatively flat domains. Figure 4(a) shows a typical atomically resolved STM image after denoising with a wavelet-based filter [12]. The image indicates the presence of carbon atoms arranged in hexagonal pattern (consistent for a short range) with a lattice constant of $0.25 \pm 0.012 \mathrm{~nm}$, agreeing well with the expected $0.246 \mathrm{~nm}$ lattice spacing of HOPG. The observed lattice is expected from the $\mathrm{AB}$ stacking of graphene sheets as in HOPG. Less well resolved lines labeled A, B and C may indicate defect/distortions in such films. Possible lattice distortions are also observed in the lower left part of the image. The features and detailed characterizations of structural disorder are to be investigated in further studies. Figure 4(b) indicates atomically resolved images of HOPG for comparison with that of graphene film on $\mathrm{SiO}_{2}$. Figure 4(b) also demonstrate that long range hexagonal pattern of carbon atoms is stable and maintained to preserve three fold symmetry which is expected from HOPG. Atomically resolved STM images are taken at a constant set point current $0.5 \mathrm{nA}$ with a bias voltage of $+0.5 \mathrm{~V}$. 

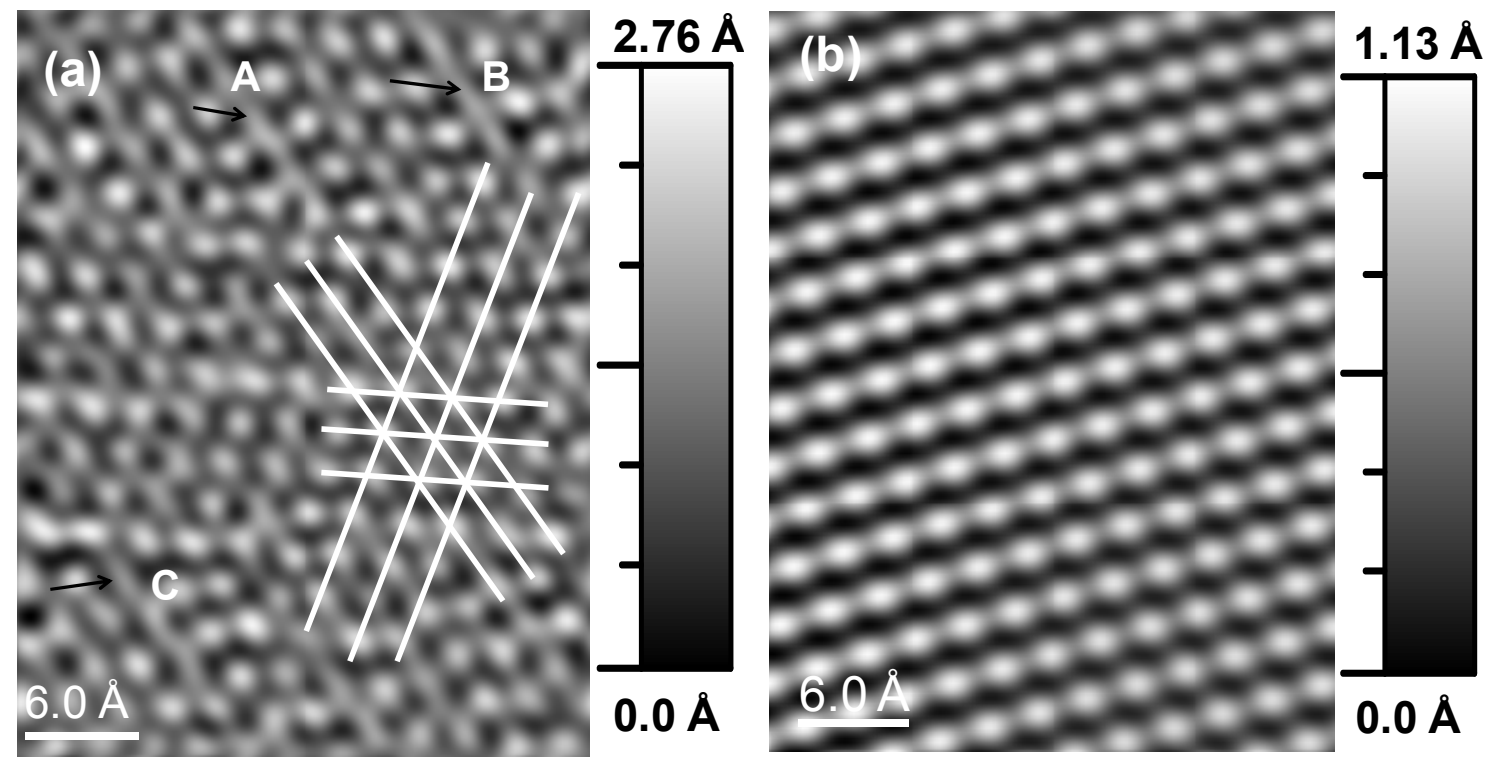

Figure 4. Atomically resolved STM images. (a) Atomically resolved images of a transferred graphene film on $\mathrm{SiO}_{2}$, indicating the presence of carbon atoms in hexagonal pattern that only exist in a relative short range. Lines labeled A, B and C may indicate defect/distortions in such films. Possible lattice distortions are also observed in the lower left part of the image. (b) Atomically resolved image of HOPG indicating the presence of carbon atoms in hexagonal pattern. The distance between two neighboring carbon atoms of graphene on $\mathrm{SiO}_{2}$ in (a) is $0.25 \pm 0.012 \mathrm{~nm}$ that matches well with that of HOPG.

\section{Acknowledgments}

We acknowledge support from Miller Family Endowment, Birck Director's Fund and Semiconductor Research Corporation (SRC)'s Nanoelectronics Research Initiative (NRI) via Midwest Institute for Nanoelectronics Discovery (MIND). Acknowledgment is also made to the donors of the American Chemical Society Petroleum Research Fund for partial support of this research. QY acknowledges support by NSF Grant 0620906 and CAM Special Funding. We thank Prof. Ron Reifenberger for helpful discussions.

\section{References}

1. A.K. Geim and K.S. Novoselov, Nature Mat. 6, 183-191 (2007).

2. R. Ruoff, Nature Nanotech. 3, 10-11 (2008).

3. C. Oshima and A. Nagashima, J. Phys. Cond. Mat. 9, 1-20 (1997).

4. Q. Yu et al., Appl. Phys. Lett. 93, 113103 (2008)

5. A. Reina et al., Nano Lett. 9, 30-35 (2009)

6. K.S. Kim et al., Nature 457, 706-710 (2009).

7. H. Cao et al., arXiv:0901:1136 (2009)

8. I. Horcas et al., Rev. Sci. Instrum. 78, 013705 (2007)

9. T. G. Kollie, Phys. Rev. B 16, 4872 (1977).

10. D. K. L. Tsang et al., Carbon 43, 2902 (2005).

11. H.A. Mizes, S.-i. Park and W.A. Harrison, Phys. Rev. B 36, 4491 (1987).

12. C. Gackenheimer, L. Cayon and R.Relfenberger, Ultramicroscopy 106, 389 (2006). 